Article

\title{
Pore Forming Properties of Cecropin-Melittin Hybrid Peptide in a Natural Membrane
}

\author{
Alberto Milani, Mascia Benedusi, Marco Aquila and Giorgio Rispoli * \\ Dipartimento di Biologia ed Evoluzione, Sezione di Fisiologia e Biofisica, National Institute of \\ Neuroscience and Neuroscience Center, Università di Ferrara, Via L. Borsari 46, I-44100 Ferrara, Italy; \\ E-Mails: alberto.milani@student.unife.it (A.M.); bndmsc@unife.it (M.B.); \\ marco.aquila@student.unife.it (M.A.)
}

* Author to whom correspondence should be addressed; E-Mail: rsg@unife.it; Tel./Fax: +39-0532-455462.

Received: 2 November 2009; in revised form: 4 December 2009 / Accepted: 10 December 2009 / Published: 11 December 2009

\begin{abstract}
The pore forming properties of synthetic cecropin-melittin hybrid peptide (Acetyl-KWKLFKKIGAVLKVL-CONH 2 ; CM15) were investigated by using photoreceptor rod outer segments (OS) isolated from frog retinae obtained by using the whole-cell configuration of the patch-clamp technique. CM15 was applied (and removed) to (from) the OS in $\sim 50 \mathrm{~ms}$ with a computer-controlled microperfusion system. Once the main OS endogenous conductance was blocked with light, the OS membrane resistance was $\geq 1 \mathrm{G} \Omega$, allowing high resolution, low-noise recordings. Different to alamethicines, CM15 produced voltage-independent membrane permeabilisation, repetitive peptide application caused a progressive permeabilisation increase, and no single-channel events were detected at low peptide concentrations. Collectively, these results indicate a toroidal mechanism of pore formation by CM15.
\end{abstract}

Keywords: peptide antibiotics; pore-forming toxins; photoreceptors; ion channels; patch clamp 


\section{Introduction}

The antimicrobial peptides form an essential part of the innate immunity against pathogens; many peptides induce bacterial death by permeabilising their plasma membrane to the point of inducing cell lyses. This permeabilisation occurs following the adhesion of the peptides parallel to the bacterial lipid bilayer, the peptide orientation perpendicular to the membrane, and finally to their insertion into the bilayer, forming transmembrane pores. Because of their amino-acid composition, amphipathicity, and cationic charge, three distinct mechanisms have been proposed to explain membrane permeabilisation: "barrel-stave", "carpet", and "toroidal-pore" (Figure 1; reviewed in [1]).

Figure 1. Mechanisms of cell membrane permeabilisation induced by a peptide. After adhering on the external face of the membrane (top), the peptide could insert in the membrane according to a barrel-stave (left), toroidal (two consecutive center diagrams), or carpet mechanism (right diagrams).

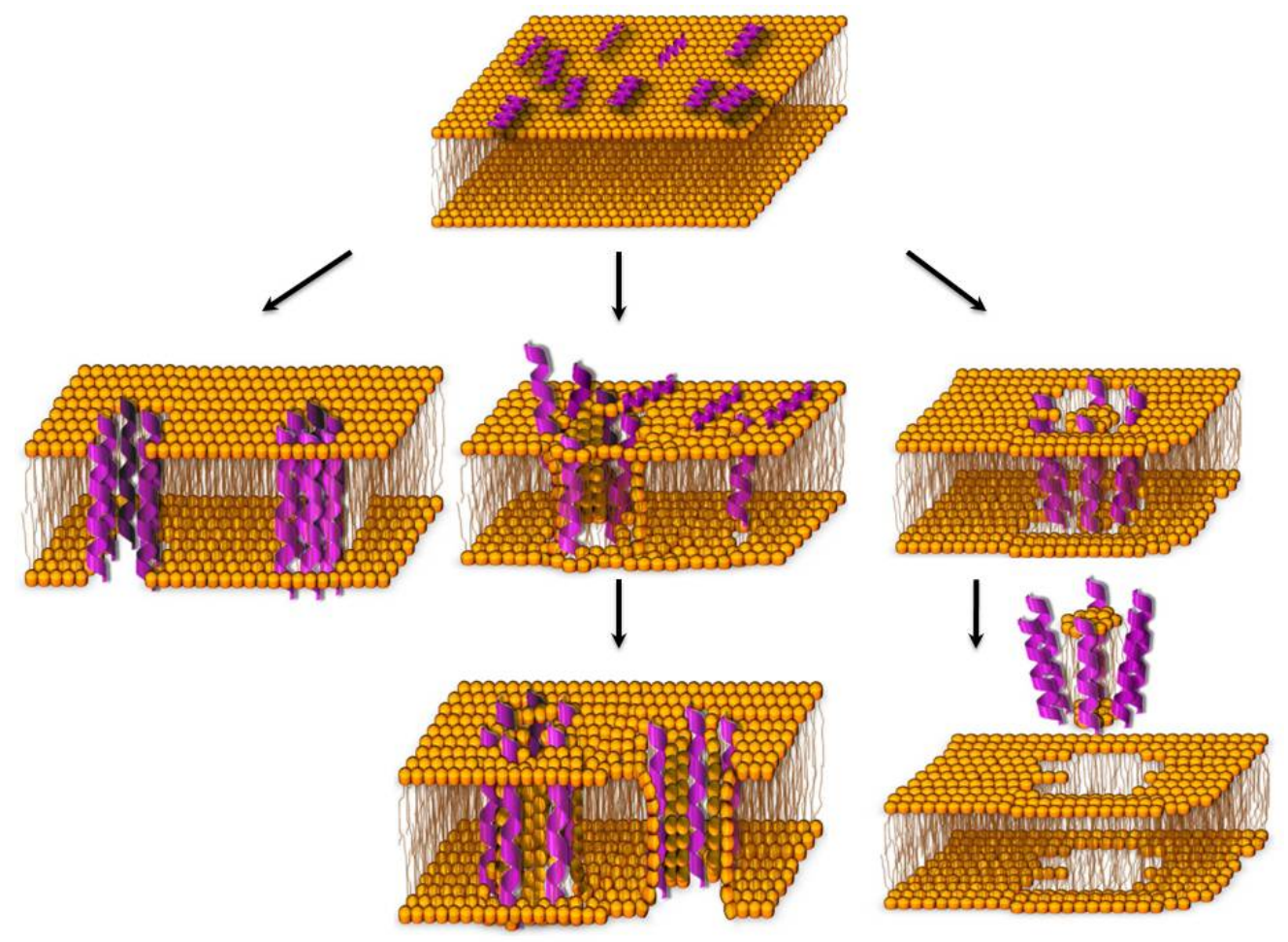

Briefly, the barrel-stave mechanism requires that peptides bind together (as staves) around a central lumen, forming a pore (the barrel); the peptide hydrophobic segments align with the lipid core region of the bilayer, while their hydrophilic segments face the lumen interior. In the toroidal-pore, the polar segments of the peptides associate with the polar head groups of the lipids so that the lipids are forced to tilt from the lamellar normal up to connect the two leaflets of the membrane, forming a continuous bend from one side to the other of the membrane, in the fashion of a toroidal hole. Differently from the barrel-stave mechanism, the peptides are always associated with the lipid head groups, even when they are perpendicularly inserted in the lipid bilayer. In the carpet mechanism, the peptides accumulated on the bilayer surface are attracted by Coulomb forces to the anionic phospholipid head groups at several sites, covering the membrane surface in a carpet-like manner. The peptides then assemble with the 
lipids to form transiently toroidal pores, allowing more and more peptides to access the membrane. Eventually, the surface-oriented peptides lead to the bilayer disintegration in a detergent-like manner, by forming micelles. It is, however, conceivable that these three mechanisms of membrane permeabilisation are not completely different and independent: for instance, it is possible that a certain peptide may act preferentially with one mechanism at a certain concentration and with another mechanism at a higher concentration [1], or in the presence of some other endogenous or exogenous molecules.

A small 15-residue synthetic hybrid peptide (CM15; see Experimental Section), first described by [2], composed of the first seven residues of the silk moth cecropin A and residues 2-9 from the bee venom peptide melittin, displays potent broad-spectrum antimicrobial activity, but seems to lack the haemolytic properties associated with melittin. It is not clear whether CM15 forms discrete membrane pores or disrupts membranes via a detergent-like carpet mechanism [2-5]. In this paper, a novel strategy has been employed to precisely assess the biophysical characteristics and the pore formation dynamics of CM15 under physiological conditions. Briefly, a custom-made, computer controlled microperfusion system was employed to rapidly apply (and remove) natural and custom-made peptides onto a cell, recorded in voltage-clamp, whole-cell configuration, in which all the endogenous conductances were fully blocked [6]. Peptide insertion dynamics was therefore given by the time course of the exogenous current at a given potential $\left(V_{h}\right)$. It has been found that the isolated rod outer segment (OS) [7] was particularly suitable to carry on the above studies, because it was possible to easily block all the endogenous currents without using any drug (such as tetrodotoxin, tetraethylammonium, dihydropyridines, etc.), that could aspecifically interfere with the pore formed by the peptides as well. The computer controlled microperfusion system, developed in collaboration with an industrial partner (De Angelis s.r.1., Genoa, Italy; Figure 2), allowed us to apply and remove ions, drugs and peptides on the cells in $\sim 50 \mathrm{~ms}$ with an accurately controlled timing. This allowed to study, besides the ion channel characteristics (such as the presence of gating, its voltage dependence, the unitary conductance, and the selectivity and blockade [8]), the dynamics of pore formation as well, that are of key importance to assess the channel performance and its potential biotherapeutic activity.

\section{Results and Discussion}

To study the biophysical properties of a pore-forming peptide inserted in a natural membrane, it was planned to extracellularly perfuse the peptides on a cell where all the endogenous sources of membrane current could be readily blocked. The photoreceptor rod outer segments (OS) mechanically isolated from Rana esculenta have been found particularly suitable to carry on this study, because of their large size (Figure 2, Right panel) and for the commercial availability and low cost of this frog species. The vertebrate OS possesses just two endogenous conductances: the light sensitive channels and the $\mathrm{Na}^{+}: \mathrm{Ca}^{2+}, \mathrm{K}^{+}$exchanger (reviewed in $[9,10]$ ). If the OS is illuminated, the light sensitive channels close; moreover, the exchanger can be blocked if just one of the ion species transported by it (i.e., $\mathrm{Na}^{+}$, $\mathrm{Ca}^{2+}$ or $\mathrm{K}^{+}$) is removed from both sides of the membrane $[11,12]$. 
Figure 2. The technique employed to investigate the permeabilisation properties of the peptides inserted in a natural membrane. Left panel, isolated rod outer segment (OS) recorded in whole-cell mode (visible on the right-side of this microphotograph) aligned in front of the multibarreled perfusion pipette (visible on the left-side; scale bar is $500 \mu \mathrm{m}$; horizontal red arrows denote perfusion flows); Right panel, the same OS at higher magnification (scale bar is $20 \mu \mathrm{m}$ ).

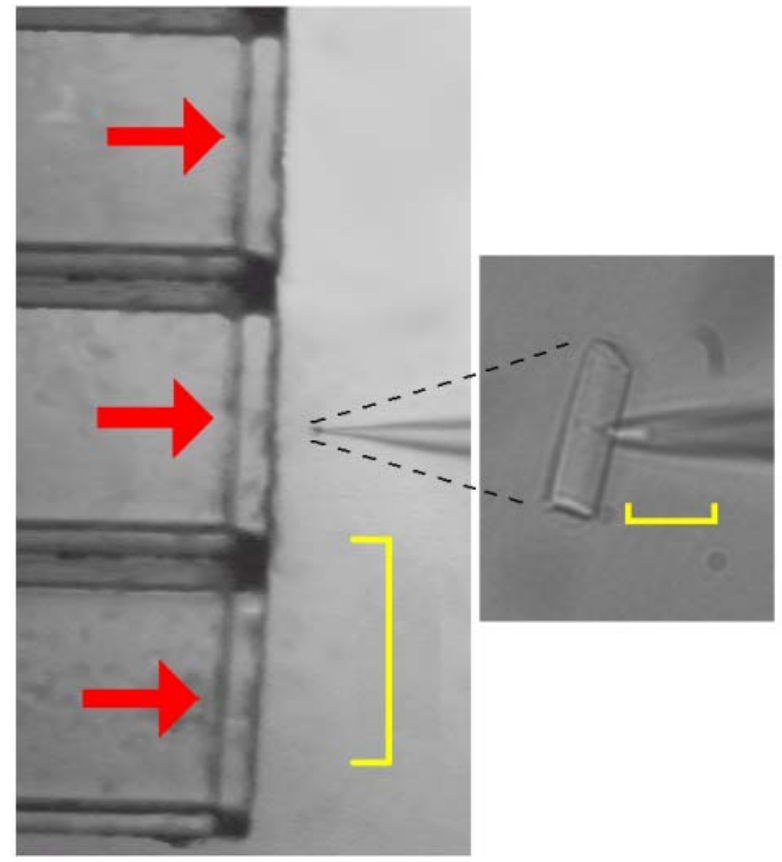

To simplify the interpretation of the experiments, patch pipettes were filled with the same perfusion solution (that typically contained $130 \mathrm{mM}$ of $\mathrm{KCl}$ ) to ensure the current was only driven by the holding potential ( $V_{h}$, usually set to $-20 \mathrm{mV}$ ). Under these ionic conditions and under room lights (that will close all the light-regulated channels), the OS membrane resistance $\left(R_{m}\right)$ was usually larger than $1 \mathrm{G} \Omega$ in the absence of the peptide, exhibiting a linear (ohmic) current-to-voltage characteristics [6]. This high $R_{m}$ value allowed current recordings with a resolution of $1 \mathrm{pA}$ in a bandwidth of at least $1 \mathrm{kHz}$; however, to preserve the membrane integrity during long recordings, it was necessary to include a physiological concentration of $\mathrm{Ca}^{2+}(1 \mathrm{mM})$ to the external solution. Therefore, $1 \mathrm{mM} \mathrm{Ca}^{2+}$ was added to the intracellular solution as well, to ensure that the current was still entirely driven by $V_{h}$. The dynamics of the pore formation was tested by means of the following protocol (Figure 3 ). With the isolated OS continuously held to $V_{h}, R_{m}$ was measured before peptide perfusion by means of a brief $-10 \mathrm{mV}$ step; the peptide was then quickly applied (in about $\sim 50 \mathrm{~ms}$ ) using the fast perfusion system. Once the current had stabilised, the OS was finally returned to the control solution (without the peptide) to assess a possible recovery of the current, and $R_{m}$ was again measured. Concentrations of CM $15 \leq 1 \mu \mathrm{M}$ gave no detectable macroscopic currents nor single channel events, in contrast to alamethicin [6], that generated clear single channel current at concentrations $\leq 250 \mathrm{nM}$. Macroscopic currents were routinely obtained at $\mathbf{C M 1 5}$ concentrations $\geq 2.5 \mu \mathrm{M}$; repetitive peptide applications produced currents of increasingly amplitude (Figures 4 and 5). These observations exclude the fact that CM15 permeabilises the plasma membrane according to a barrel-stave mechanism. The latter requires 
that a certain number of monomers binds together once in the plasma membrane to form an ion conductive pore: if the peptide concentration is small, then pores are formed and disaggregated frequently, producing single channel events; at higher concentrations, membrane peptides equilibrate with the ones externally perfused, giving a stable macroscopic current $[6,13]$. The increase in plasma membrane permeability to ions induced by CM15 can be described quantitatively by the following kinetic parameters:

The activation delay $\left(D_{d}\right)$, defined as the time lag between peptide application and the time in which the current deviates from its baseline (following peptide application), more than three times the noise average fluctuation (indicated by the green arrow in the Figure 3, Inset);

The activation time constant $\left(\tau_{a}\right)$, defined as the time constant of the single exponential fit to current activation (Figure 3);

The current amplitude at steady-state $\left(I_{\max }\right)$;

The deactivation delay $\left(D_{d}\right)$, defined as the time lag between peptide removal and the time in which the current deviates from its baseline more than three times the noise average fluctuation;

The deactivation time constant $\left(\tau_{d}\right)$ defined as the time constant of the single exponential fit to current deactivation (Figure 3).

Figure 3. Kinetics of OS membrane permeabilisation induced by $2.5 \mu \mathrm{M}$ concentration of CM15. Red bar indicate the timing of the peptide application and withdrawal; green traces are exponential fittings of the activation and deactivation phases of the current $\left(\tau_{a} \approx 7 \mathrm{~s}\right.$; $\tau_{d} \approx 8.5 \mathrm{~s} ; I_{\max } \approx-580 \mathrm{pA} ; V_{h}=-20 \mathrm{mV}$ ); blue arrows are $700 \mathrm{~ms}$ voltage steps of $-10 \mathrm{mV}$ superimposed to $V_{h}$ to measure $R_{m}$ (that was $1 \mathrm{G} \Omega$ before peptide application and $200 \mathrm{M} \Omega$ within $50 \mathrm{~s}$ after peptide removal from the external perfusion solution). Inset: initial activation phase of the current at high resolution; red bar indicate the timing of peptide application $\left(D_{a} \approx 1.0 \mathrm{~s}\right.$, indicated by the green arrow; $\left.D_{d} \approx 0.5 \mathrm{~s}\right)$.

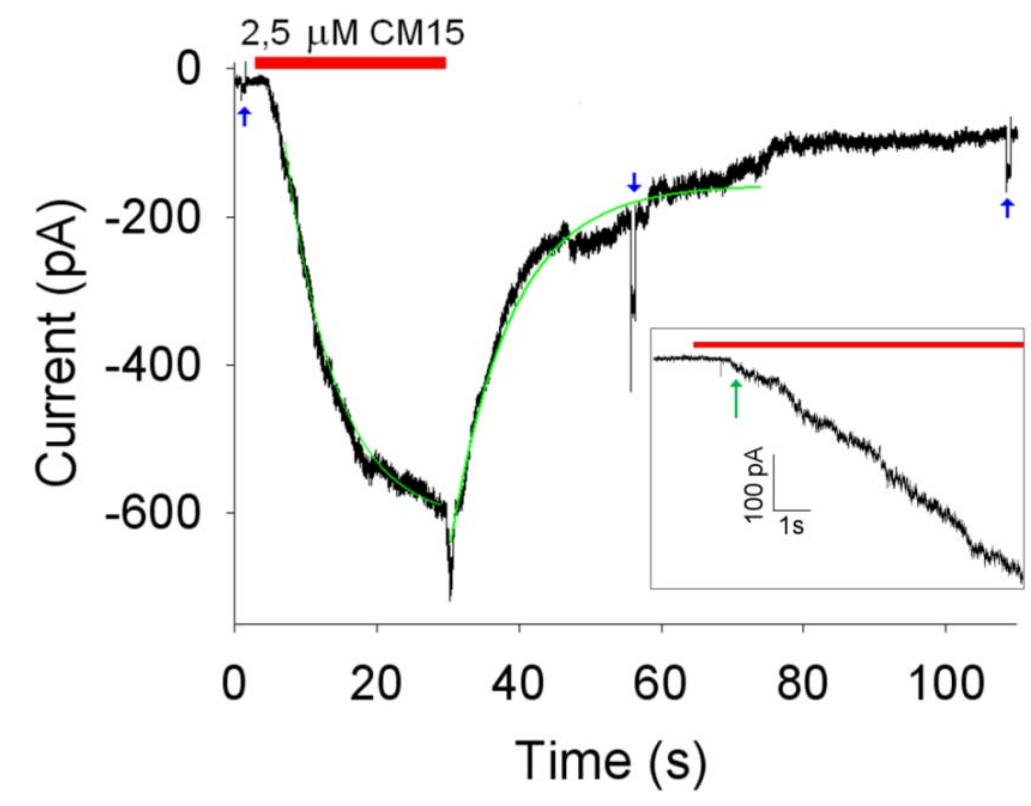

However, these parameters cannot be unambiguously estimated, since they may change as the current increases (compare, for instance, $\tau_{a}$ at 2.5 and $10 \mu \mathrm{M}$ of CM15 reported in the captions of 
Figures 3 and 4). To obtain a reproducible value of these parameters, recordings were selected to approximately give current amplitudes between 400 and $600 \mathrm{pA}$, irrespective of the peptide concentration. A representative example of one of these recordings is shown in Figure 3; the average value of the kinetics parameters were: $D_{a} \approx 0.8 \pm 0.2 \mathrm{~s} ; D_{d} \approx 0.6 \pm 0.2 \mathrm{~s} ; \tau_{a} \approx 8.4 \pm 1.4 \mathrm{~s} ; \tau_{d} \approx 10 \pm 2 \mathrm{~s}$; $I_{\text {max }} \approx-510 \pm 51 \mathrm{pA}\left(V_{h}=-20 \mathrm{mV} ; n=6\right)$. The largest values of the kinetics parameter were measured by using the CM15 at $10 \mu \mathrm{M}$ concentration; larger concentrations were not used to avoid the loss of voltage control. Indeed, as the current increases, there is a voltage error induced by the access resistance (that was typically $\sim 15 \mathrm{M} \Omega$ in the recordings considered in this paper) that can be as high as $15 \mathrm{mV}$ at $1 \mathrm{nA}$ of current.

Figure 4. Kinetics of OS membrane permeabilisation induced by $10 \mu \mathrm{M}$ concentration of CM15. Red bars indicate peptide application and withdrawal timing; blue arrows indicate the $-10 \mathrm{mV}$ steps used to measure $R_{m}$ before $(\sim 200 \mathrm{M} \Omega)$ and at the steady state after peptide removal $(\sim 120 \mathrm{M} \Omega$ following the first application and $\sim 45 \mathrm{M} \Omega$ following the second one). Kinetics parameter relative to the second application (where the current was maximal): $D_{a} \approx 0.6 \mathrm{~s} ; D_{d} \approx 0.4 \mathrm{~s} ; \tau_{a} \approx 1.1 \mathrm{~s} ; \tau_{d} \approx 2.7 \mathrm{~s} ; I_{\max } \approx-930 \mathrm{pA}$.

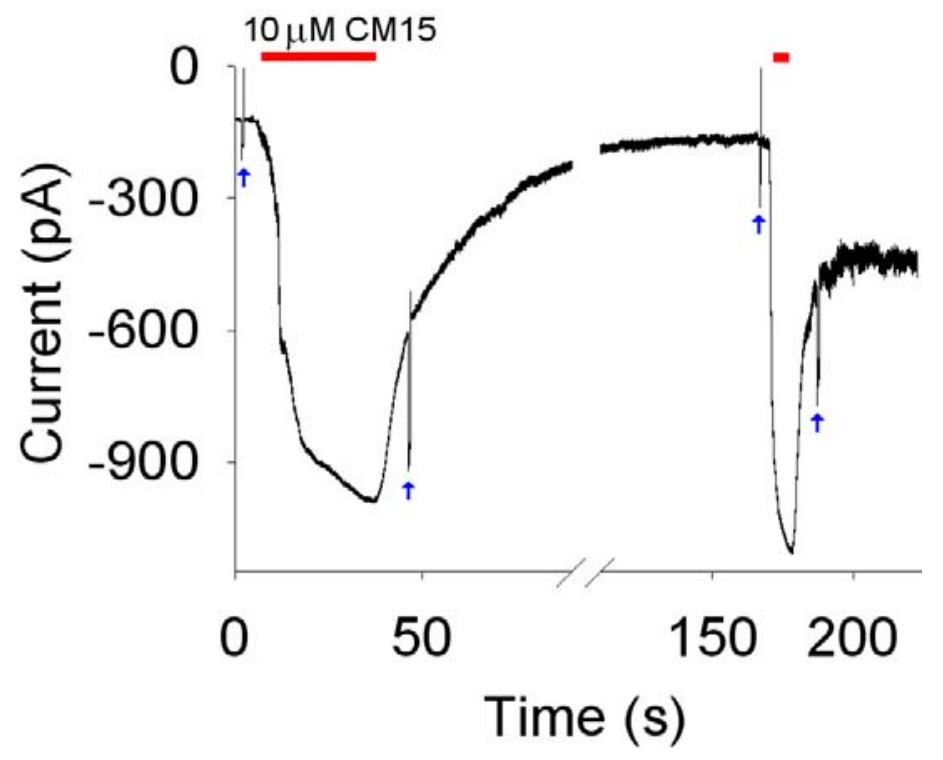

In contrast to alamethicin, the current did not return to the zero level following peptide removal $[6,13]$ : the current recovered instead to a plateau level and the $R_{m}$ measured on this plateau was consequently smaller in respect to the one measured before the CM15 application. The CM15 concentration was larger, and/or more and more applications were performed (i.e., the larger was the current induced by CM15), the larger was the plateau amplitude and the smaller was the $R_{m}$ (measured during the plateau phase). The incomplete recovery in respect to alamethicin and the progressive current increase observed with repetitive CM15 applications indicate that CM15 makes pores that are relatively stable and that are larger and larger and/or more and more numerous with repetitive applications. However, the substantial recovery of current and the cell integrity, observed even at high CM15 concentrations (Figure 4), exclude the carpet mechanism of membrane permeabilisation: micellation is expected to be severe under these conditions, producing the irreversible disruption of the membrane and cell lyses. Therefore, it can be concluded that CM15 permeabilises the membrane 
according to a toroidal mechanism of pore formation. This view is also supported by the voltageindependency of CM15 membrane permeabilisation, in contrast to alamethicin, that instead inserts in the OS membrane at negative voltages [6,13]. Indeed, CM15 application at $+20 \mathrm{mV}$ or $-20 \mathrm{mV}$ produced currents with similar $I_{\max }$ (and $D_{a}, D_{d}, \tau_{a}, \tau_{d}$ as well; Figure 5, Left panel). However, the latter protocol is not suitable to assess the precise voltage dependency of the current, since the current progressively increases with repetitive application of CM15 (note that the maximal current amplitude at $+20 \mathrm{mV}$ in Figure 5 is larger in respect to the one recorded at $-20 \mathrm{mV}$ ). To circumvent this problem, rapid voltage ramps (slope: $0.25 \mathrm{mV} / \mathrm{ms}$ ) were applied during CM15 perfusion at $V_{h}=-20 \mathrm{mV}$, waiting for the current to stabilise for a period at least as long as the ramp (400 ms). To avoid the loss of voltage control at extreme voltages $(-60$ and $+40 \mathrm{mV}$; see above), cells were selected to have currents of about $100-200 \mathrm{pA}$ at $V_{h}=-20 \mathrm{mV}$. The resulting current to voltage relationship was almost perfectly ohmic for physiological voltages (Figure 5, Right panel) in all cells examined $(n=4)$.

Figure 5. Voltage dependency of membrane permeabilisation induced by CM15. Left panel, $5 \mu \mathrm{M}$ of CM15 applied at $V_{h}=-20 \mathrm{mV}$ and $+20 \mathrm{mV}$; Right panel, current to voltage relationship obtained using a voltage ramp on a different cell (see text) when current attained a plateau (-97 pA at $V_{h}=-20 \mathrm{mV}$ ) during perfusion with $2.5 \mu \mathrm{M}$ of CM15. Blue arrows indicate the $-10 \mathrm{mV}$ steps used to measure $R_{m}$.

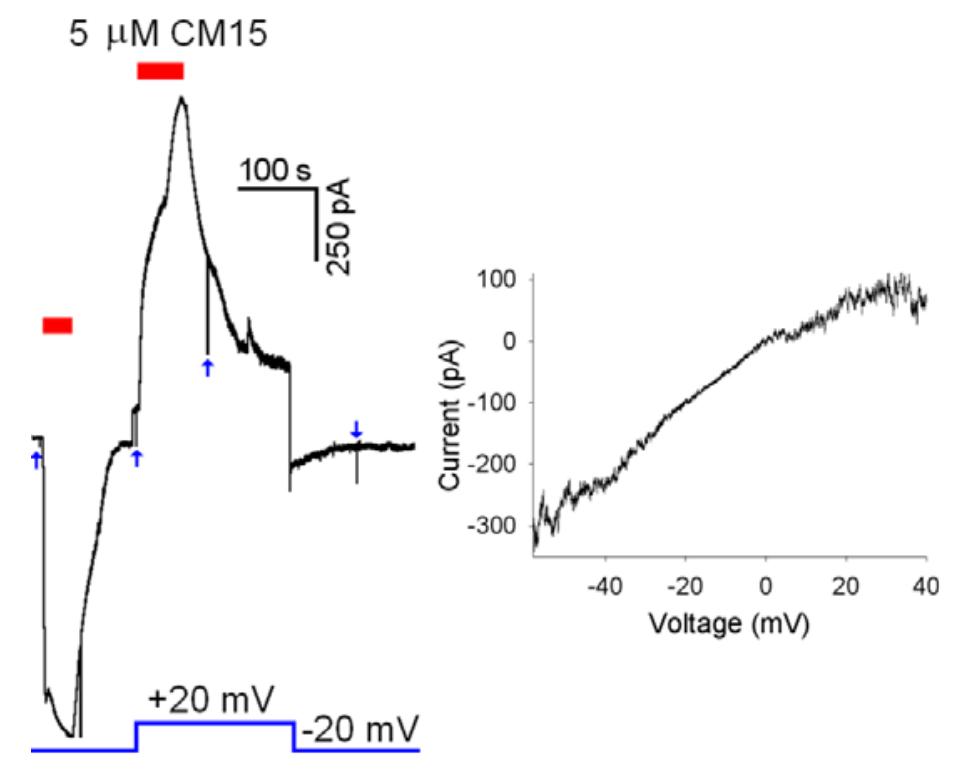

Remarkably, the aminoacids of CM15 must be lined up in a precise sequence to produce efficient membrane permeabilisation: indeed, a random sequence of these aminoacids, like the one reported in the Experimental section (scrambled CM15), is not able to produce any permeabilisation, even for repetitive applications at $10 \mu \mathrm{M}$ concentration (lasting up to $3 \mathrm{~min} ; n=3 \mathrm{OS}$; data not shown).

\section{Experimental Section}

Rod outer segments (OS) were mechanically isolated from the retina of the Rana esculenta. Methods are described in detail elsewhere [6]. Animal experiments and care were performed in compliance with the Declaration of Helsinki guidelines and a local ethical Committee approved the experimental procedures. All manipulations were made in the dark using infrared illumination and an 
infrared viewer (Find-R-Scope, FJW Optical Systems, Palatine, IL, USA). Before dissection, the animal was dark adapted $(\approx 4 \mathrm{hr})$, anaesthetized by immersion in a tricaine methanesulphonate solution ( $1 \mathrm{~g} / \mathrm{L}$ in water), and then decapitated. Both eyes were removed from the head and hemisected. The back half of the eyeball was cut into pieces (up to four) that were stored in oxygenated Ringer solution on ice and used when needed. The retina was "peeled" from an eyecup piece and was gently triturated in Ringer $(\sim 5 \mathrm{~mL})$, using a fire-polished Pasteur pipette to obtain the OS. A fluid drop containing the OS was then transferred to the recording chamber. The OS on the microscope stage (TE 300, Nikon, Tokyo, Japan) were illuminated with an ultrabright infrared LED $(900 \mathrm{~nm})$ and viewed in a window of AquaCosmos software package (version 2.5.3.0; Hamamatsu Photonics, Tokyo, Japan), controlling via a PCI board (PCDIG, Dalsa, Waterloo, ON, Canada) a fast digital camera (C6790-81, Hamamatsu Photonics) coupled to the microscope.

OS were recorded using the whole-cell configuration of the patch-clamp technique under visual control at room temperature $\left(20-22{ }^{\circ} \mathrm{C}\right.$; Figure 2). The Ringer solution had the following composition (in $\mathrm{mM}$ ): $115 \mathrm{NaCl}, 3 \mathrm{KCl}, 10$ HEPES free acid [ $N$-(2-hydroxyethyl)piperazine- $N$ '-(2-ethanesulfonic acid)], $0.6 \mathrm{MgCl}_{2}, 0.6 \mathrm{MgSO}_{4}, 1.5 \mathrm{CaCl}_{2}, 10$ glucose (osmolality $260 \mathrm{mOsm} / \mathrm{Kg}$, buffered to $\mathrm{pH}=7.6$ ). All chemicals were purchased from Sigma (St. Louis, MO, USA).

The current amplitude (recorded employing an Axopatch 200B; Molecular Devices, Sunnyvale, CA, USA) elicited by a $-10 \mathrm{mV}$ pulse in cell attached and during whole-cell recording was used to measure seal resistance and $R_{m}$, respectively. Once the whole cell recording was obtained, the current transients elicited by $-10 \mathrm{mV}$ voltage pulses were used to measure access resistance and cell capacitance; the cell was then aligned in front of a multibarrelled perfusion pipette that can be moved on an horizontal plane (Figure 2, Left panel). Peptides were applied and removed in $\sim 50$ ms by switching forth and back the OS from a stream of control perfusion solution [composition (in $\mathrm{mM}$ ): $130 \mathrm{~K}^{+}, 1 \mathrm{Ca}^{2+}$ and $10 \mathrm{HEPES}$; osmolality $260 \mathrm{mOsm} / \mathrm{Kg}$, buffered to $\mathrm{pH}=7.6$ with $\mathrm{KOH}$ ] to a stream containing the peptide (dissolved in the same perfusion solution). This strategy allowed us to assess the dynamics of the pore formation and the possible reversibility of this process [6]. Patch pipettes were filled with the same perfusion solution in order to drive the current just with the $V_{h}$ (that was typically $-20 \mathrm{mV})$.

The primary structure of CM15 peptide was: acetyl-KWKLFKKIGAVLKVL-CONH 2 ; as a control, to ensure that the CM15-induced permeabilisation was not due to some aspecific effect produced by the interaction of one or more of its aminoacids with some membrane protein, a "scrambled" version of CM15 was tested (primary structure: acetyl-KWKLKFKIGLVKLVAV-NH ${ }_{2}$ ). Both peptides were a generous gift of Dr. Feix (of Department of Biophysics, Medical College of Wisconsin, Milwaukee, WI, USA). CM15 and its scrambled version were dissolved in bidistilled water to get a $500 \mu \mathrm{M}$ stock solution; an aliquot of this stock was dissolved in the perfusion solution to get a final concentration of $1,2.5,5$ and $10 \mu \mathrm{M}$, and used within $30 \mathrm{~min}$.

Recordings were filtered at $2 \mathrm{kHz}$ via an eight-pole Butterworth filter (VBF/8 Kemo, Beckenham, UK), sampled on-line at $5 \mathrm{kHz}$ by a Digidata 1322A (Molecular Devices) connected to the SCSI port of a Pentium computer running the pClamp 9.0 software package (Molecular Devices), and stored on disk. Data were further low-pass filtered off-line at $200 \mathrm{~Hz}$ using a Gaussian filter and analyzed using Clampfit (version 9.0; Molecular Devices). Figures and statistics were performed using SigmaPlot (version 8.0; Jandel Scientific, San Rafael, CA, USA). Results are given as means \pm SEM. 


\section{Conclusions}

The cecropin-melittin hybrid peptide CM15 produced voltage-independent permeabilisation of photoreceptor rod outer segment (OS) membranes. Repetitive peptide applications at concentrations $>2 \mu \mathrm{M}$ caused the progressive increase of the steady-state current amplitude; no discernible singlechannel events were detected at low peptide concentrations (i.e., $\leq 1 \mu \mathrm{M}$ ), thus excluding a barrel-stave mechanism of membrane permeabilisation. The cell integrity and the substantial reversibility of permeabilisation observed at concentrations as high as $10 \mu \mathrm{M}$ of CM15 would not be expected in the case of a carpet mechanism of pore formation, because at these concentrations the micellation is expected to disrupt the OS membrane causing its lyses. Collectively, these results indicate that CM15 inserts in the plasma membrane according to a toroidal mechanism of pore formation.

\section{Acknowledgements}

We thank Martina Infanti for her invaluable help with some experiments, Andrea Margutti for technical assistance, and Jimmy B. Feix (of Department of Biophysics, Medical College of Wisconsin, Milwaukee, WI, USA) for providing the CM15 and its scrambled version. Anna Fasoli and Cristina Mantovani helped with data analysis and experiment preparation. Financial support to G.R. included grants from the Ministero dell'Università e della Ricerca (MIUR), Roma (Italy) and from the "Comitato dei sostenitori dell'Università di Ferrara" (Project FAR: Correnti ioniche nei peptidi antibiotici e virali e nelle cellule sensoriali e gangliari, Ferrara, Italy).

\section{References}

1. Brogden, K.A. Antimicrobial peptides: Pore formers or metabolic inhibitors in bacteria? Nat. Rev. Microbiol. 2005, 3, 238-250.

2. Andreu, D.; Ubach, J.; Boman, A.; Wahlin, B.; Wade, D.; Merrifield, R.B.; Boman, H.G. Shortened cecropin A-melittin hybrids. Significant size reduction retains potent antibiotic activity. FEBS Lett. 1992, 296, 190-194.

3. Juvvadi, P.; Vunnam, S.; Merrifield, E.L.; Boman, H.G.; Merrifield, R.B. Hydrophobic effects on antibacterial and channel-forming properties of cecropin A-melittin hybrids. J. Pept. Sci. 1996, 2 , 223-232.

4. Mancheno, J.M.; Onaderra, M.; Martinez del Pozo, A.; Diaz-Achirica, P.; Andreu, D.; Rivas, L.; Gavilanes, J.G. Release of lipid vesicle contents by an antibacterial cecropin A-melittin hybrid peptide. Biochemistry 1996, 35, 9892-9899.

5. Steiner, H.; Andreu, D.; Merrifield, R.B. Binding and action of cecropin and cecropin analogues: antibacterial peptides from insects. Biochim. Biophys. Acta 1988, 939, 260-266.

6. Vedovato, N.; Rispoli, G. A novel technique to study pore-forming peptides in a natural membrane. Eur. Biophys. J. 2007, 36, 771-778.

7. Rispoli, G.; Sather, W.A.; Detwiler, P.B. Visual transduction in dialysed detached rod outer segments from lizard retina. J. Physiol. 1993, 465, 513-537.

8. Hille, B. Ion Channels of Excitable Membranes, 3rd ed.; Sinauer: Sunderland, MA, USA, 2001. 
9. Rispoli, G. Calcium regulation of phototransduction in vertebrate rod outer segments. $J$. Photochem. Photobiol. B-Biol. 1998, 44, 1-20.

10. Moriondo, A.; Rispoli, G. A step-by-step model of phototransduction cascade shows that $\mathrm{Ca}^{2+}$ regulation of guanylate cyclase accounts only for short-term changes of photoresponse. Photochem. Photobiol. Sci. 2003, 2, 1292-1298.

11. Rispoli, G.; Navangione, A.; Vellani, V. Transport of $\mathrm{K}$ by photoreceptor $\mathrm{Na} / \mathrm{Ca}, \mathrm{K}$ exchanger in isolated rods of lizard retina. Biophys. J. 1995, 69, 74-83.

12. Vedovato, N.; Rispoli, G. Modulation of the reaction cycle of the $\mathrm{Na}(+)$ : $\mathrm{Ca}(2+), \mathrm{K}(+)$ exchanger. Eur. Biophys. J. 2007, 36, 787-793.

13. Vedovato, N.; Baldini, C.; Toniolo, C.; Rispoli, G. Pore-forming properties of alamethicin F50/5 inserted in a biological membrane. Chem. Biodivers. 2007, 4, 1338-1346.

Sample Availability: Not available.

(C) 2009 by the authors; licensee Molecular Diversity Preservation International, Basel, Switzerland. This article is an open-access article distributed under the terms and conditions of the Creative Commons Attribution license (http://creativecommons.org/licenses/by/3.0/). 